ALEA, Lat. Am. J. Probab. Math. Stat. 16, 15-31 (2019)

DOI: 10.30757/ALEA.v16-02

\title{
On the semimartingale property of Brownian bridges on complete manifolds
}

\author{
Batu Güneysu \\ Universität Bonn \\ Endenicher Allee 60, 53115 Bonn \\ E-mail address: gueneysu@math.uni-bonn.de
}

\begin{abstract}
It is shown that every adapted Brownian bridge on a geodesically complete connected Riemannian manifold is a semimartingale including its terminal time, without any further assumptions on the geometry. In particular, it follows that every such process can be horizontally lifted to a smooth principal fiber bundle with connection, including its terminal time. The proof is based on a localized Hamilton-type gradient estimate.
\end{abstract}

\section{Introduction}

Given $x, y \in \mathbb{R}^{m}, T>0$, let $\Omega_{T}\left(\mathbb{R}^{m}\right)$ stand for the Wiener space of continuous paths $\omega:[0, T] \rightarrow \mathbb{R}^{m}$. We denote with $\mathbb{P}^{x, T}$ the usual Euclidean Wiener measure (that is, the Brownian motion measure) and with $\mathbb{P}^{x, y, T}$ the usual Euclidean pinned Wiener measure (that is, the Brownian bridge measure) on $\Omega_{T}\left(\mathbb{R}^{m}\right)$ with its Borelsigma-algebra $\mathscr{F}^{T}$. Then with $X$ the coordinate process on $\Omega_{T}\left(\mathbb{R}^{m}\right)$ and $\mathscr{F}_{*}^{T}=$ $\left(\mathscr{F}_{t}^{T}\right)_{t \in[0, T]}$ the filtration of $\mathscr{F}^{T}$ that is generated by $X$, the following important fact is well-known to hold true:

(S) $\left(X_{t}\right)_{t \in[0, T]}$ is a continuous semimartingale with respect to $\left(\mathbb{P}^{x, y, T}, \mathscr{F}_{*}^{T}\right)$.

Let us point out here that, as for all $0<t<T$ one has $\left.\left.\mathbb{P}^{x, y, T}\right|_{\mathscr{F}_{t}^{T}} \sim \mathbb{P}^{x, T}\right|_{\mathscr{F}_{t}^{T}}$, the property (S) only becomes nontrivial at $t=T$. Furthermore, the importance of $(\mathrm{S})$ is already clear at a very fundamental level: Continuous disintegrations of probabilistic formulae for covariant Schrödinger semigroups (Güneysu, 2011, 2012) clearly require such a result. We refer the reader to Güneysu (2011) for such a continuous disintegration in the Euclidean case.

In this paper we will be concerned with the validity of the semimartingale property (S) on noncompact Riemannian manifolds. To this end, we start by recalling

Received by the editors March 26, 2018; accepted December 11th, 2018.

2010 Mathematics Subject Classification. 82C44, 60K35, 60G70.

Key words and phrases. Brownian bridge, heat kernel, Riemannian manifold, semimartingale. 
that given a connected Riemannian manifold $M$ of dimension $m$, the corresponding Riemannian data $\Omega_{T}(M), \mathbb{P}^{x, y, T}, \mathbb{P}^{x, T}, X, \mathscr{F}^{T}, \mathscr{F}_{*}^{T}$, as well as the question whether one has $(\mathrm{S})$ or not still makes sense: One just has to take the minimal positive heat kernel $p\left(t, x_{1}, x_{2}\right)$ everywhere in the definition of the underlying measures, replacing the Euclidean heat kernel $t^{-m / 2} e^{-\left|x_{1}-x_{2}\right|^{2} /(4 t)}$ (cf. Definition 2.1 below), and to note that $X$ is a continuous $M$-valued semimartingale, if and only if $f(X)$ is a real-valued one, for all smooth functions $f: M \rightarrow \mathbb{R}$. In fact $(\mathrm{S})$, has been established quite some time ago (1984) on compact $M$ 's by Bismut (1984b), who used the resulting "covariant" continuous disintegration in his proof of the AtiyahSinger index theorem Bismut (1984a) (the reader may also wish to consult Driver, 1994 and Hsu, 2002). We refer the reader also to the recent paper Li (2016) by X.-M. Li: There, the semimartingale property of more general hypoelliptic bridges on compact manifolds is shown to hold.

Concerning (S) for noncompact $M^{\prime} s$, we point out that this property has been stated in Aida (2000) under very restrictive geometric assumptions, such as a bounded Ricci curvature plus a positive injectivity radius, and indeed there seems to be a widely spread belief that $(\mathrm{S})$ requires some global curvature bounds in order to hold true. The reason for this might be that if one follows the typical proofs from the compact case too closely, it is tempting to believe that one needs to establish the integrability

$$
\mathbb{E}^{x, y, T}\left[\int_{0}^{T}\left|d \log p(T-t, \cdot, y)\left(X_{t}\right)\right| d t\right]<\infty
$$

which in the compact case is proved using a global gradient estimate of the form

$$
\left|d \log p\left(t, \cdot x_{1}\right)\left(x_{2}\right)\right| \leq C_{T}\left(t^{-1 / 2}+t^{-1} d\left(x_{1}, x_{2}\right)\right) \quad \text { for all } x_{1}, x_{2} \in M, 0<t \leq T,
$$

an inequality that certainly requires global curvature bounds. We point out here that problems like (1.1) arise naturally in this context, as under $\left(\mathbb{P}^{\cdot}, y, T, \mathscr{F}_{*}^{T}\right)$ the process $\left.X\right|_{[0, T)}$ is a diffusion which is generated by the time-dependent differential operator

$$
\begin{aligned}
& {\left[\mathscr{A}_{t}^{y} f\right](z)=(1 / 2) \Delta f(z)-(d \log p(T-t, \cdot, y)(z), d f),} \\
& f \in C^{\infty}(M), 0<t<T, z \in M
\end{aligned}
$$

where $\Delta$ denotes the Laplace-Beltrami operator, $d F$ the differential of a function $F$ on $M$ (a 1-form on $M$ ), and $(\cdot, \cdot)$ for the induced metric on $T^{*} M$. In particular, as $p\left(t, x_{1}, x_{2}\right)$ becomes singular near $t=0$, it is clear that some upper bound on $\left|d \log p\left(t, \cdot, x_{1}\right)\left(x_{2}\right)\right|$ for small $t$ has to be established in any case, where $|\cdot|=(\cdot, \cdot)^{1 / 2}$. It is also instructive to note that in the Euclidean case one has

$$
\begin{aligned}
& {\left[\mathscr{A}_{t}^{y} f\right](z)=(1 / 2) \Delta f(z)+(1 / 2)(T-t)^{-1} \sum_{i=1}^{m}\left(y^{i}-z^{i}\right) \partial_{i} f(z),} \\
& z=\left(z^{1}, \ldots, z^{m}\right) \in \mathbb{R}^{m} .
\end{aligned}
$$

In this paper, we are going to prove that $(S)$ holds true on every geodesically complete connected Riemannian manifold, without any further curvature assumptions. 
In fact, this will be a consequence of our main result Theorem 2.8 below. Let us point out that from a probabilistic point of view, the above result is very natural, as the semimartingale property of a manifold-valued continuous adapted process $X^{\prime}$ can be geometrically localized: Namely, it is easily seen that such a process is a semimartingale, if and only if $f\left(X^{\prime}\right)$ is a real-valued one, for all smooth compactly supported functions $f$ on the manifold. On the other hand, some serious technical difficulties arise on the analytic side, as the heat kernel is a very global object. As a striking example for this global behaviour Gong and Wang (2001) we would like to point out a result by F.-Z. Gong and F.-Y. Wang, which states that a smooth geodesically complete connected Riemannian manifold $M^{\prime}$ with Ricci curvature bounded from below is compact, if and only if it holds that

$$
\int_{M^{\prime}} p^{\prime}(t, x, y)^{-1} d \mu^{\prime}(y)<\infty \quad \text { for some } t>0, x \in M^{\prime} .
$$

Confirming the global behaviour of the heat kernel, the analysis of our paper shows that the derivation of the necessary localized estimates for the heat kernel and the logarithm of its gradient is indeed a rather delicate business. In this context, using a highly subtle local parabolic gradient bound from Arnaudon and Thalmaier (2010), we are able to prove the following inequality: In Theorem 2.9 it is shown that for every $z_{0} \in M, R_{0}>0$ there exists a constant $C>0$ which depends on the geometry of $M$ in a neighbourhood of $B\left(z_{0}, R_{0}\right)$, such that for all

$$
\left(t, x_{1}, x_{2}\right) \in\left(0, R_{0}^{2}\right] \times B\left(z_{0}, R_{0}\right) \times B\left(z_{0}, R_{0}\right)
$$

one has

$$
\left|d \log p\left(t, \cdot, x_{1}\right)\left(x_{2}\right)\right| \leq C\left(t^{-1 / 2}+t^{-1} d\left(x_{1}, x_{2}\right)\right) .
$$

The latter localized bound turns out to be enough to establish a localized version of (1.1) and ultimately (S).

Finally, we would like to add that in fact our main result Theorem 2.8 is more general than $(\mathrm{S})$ in the following sense: We define (cf. Definition 2.3 below) an arbitrary adapted continuous stochastic process

$$
X^{x, y, T}:[0, T] \times\left(\Omega, \mathscr{F},\left(\mathscr{F}_{t}\right)_{t \in[0, T]}, \mathbb{P}\right) \longrightarrow M
$$

to be an adapted Brownian bridge from $x$ to $y$ at the time $T$, if the law of $X^{x, y, T}$ is equal to $\mathbb{P}^{x, y, T}$ and if $X^{x, y, T}$ has a certain time-inhomogeneous Markov property. In case $\left(\mathscr{F}_{t}\right)_{t \in[0, T]}=\left(\mathscr{F}_{t}^{X^{x, y, T}}\right)_{t \in[0, T]}$ is the filtration generated by $X^{x, y, T}$, then this Markov property is automatically satisfied (cf. Lemma 2.4 below). In this context, our main result Theorem 2.8 states that in fact every adapted Brownian bridge is a continuous semimartingale. In particular, this result entails that (cf. Corollary 2.11 below) every adapted Brownian bridge on a geodesically complete Riemannian manifold can be horizontally lifted to principal bundles that are equipped with a connection.

\section{Main results}

In the sequel, we understand our function spaces to be real-valued. Let $M \equiv$ $(M, g)$ be a smooth connected Riemannian $m$-manifold, with $\Delta$ its Laplace-Beltrami operator. Let $d(x, y)$ denote the geodesic distance, and $B(x, r)$ corresponding open 
balls. We denote with $p(t, x, y), t>0, x, y \in M$, the minimal nonnegative heat kernel on $M$, that is, for each fixed $y$, the function $p(\cdot, \cdot, y)$ is the pointwise minimal nonnegative smooth fundamental solution of the following heat equation in $(0, \infty) \times$ $M$,

$$
(\partial / \partial t-(1 / 2) \Delta) p(\cdot, \cdot, y)=0, p(t, \cdot, y) \rightarrow \delta_{y} \quad \text { as } t \rightarrow 0+,
$$

where $\Delta$ denotes the Laplace-Beltrami operator. It follows that $(t, x, y) \mapsto p(t, x, y)$ is jointly smooth, and the connectedness of $M$ implies in fact the positivity $p(t, x, y)$ $>0$. With $d \mu$ the Riemannian volume measure we define

$$
P_{t} f(z):=\int p(t, z, w) f(w) d \mu(w), \quad f \in \bigcup_{q \in[1, \infty]} L^{q}(M), z \in M, t>0 .
$$

Then $(t, z) \rightarrow P_{t} f(z)$ is smooth in $(0, \infty) \times M$ for every such $f$. We will denote with

$$
d: C^{\infty}(M) \longrightarrow \Gamma_{C^{\infty}}\left(M, T^{*} M\right)
$$

the usual differential. The smooth metric on $T^{*} M$ will be denoted with $(\cdot, \cdot)$, where $|\cdot|=(\cdot, \cdot)^{1 / 2}$ denotes the corresponding norm. With respect to the scalar product on $L^{2}(M)$ that comes from $\mu$, and the one on $\Gamma_{L^{2}}\left(M, T^{*} M\right)$ that comes from $\mu$ and $(\cdot, \cdot)$, one has $\Delta=d^{\dagger} d$.

Given $T>0$ we denote with $\Omega_{T}(M)$ the Wiener space of continuous paths $\omega:[0, T] \rightarrow M$. We give the latter the topology of uniform convergence. Let $\mathscr{F}^{T}$ denote the Borel-sigma algebra on $\Omega_{T}(M)$, and let $\mathscr{F}_{*}^{T}:=\left(\mathscr{F}_{t}^{T}\right)_{t \in[0, T]}$ denote the filtration of $\mathscr{F}^{T}$ which is generated by the underlying canonical coordinate process. Note here that $\mathscr{F}^{T}=\mathscr{F}_{T}^{T}$. The following result is well-known (cf. Bär and Pfäffle, 2011 for a detailed proof):

Proposition and definition 2.1. 1. For every $x_{0} \in M$, the Wiener measure $\mathbb{P}^{x_{0}, T}$ from $x_{0}$ with terminal time $T$ is defined to be the unique sub-probability measure on $\left(\Omega_{T}(M), \mathscr{F}^{T}\right)$ which satisfies

$$
\begin{aligned}
& \mathbb{P}^{x_{0}, T}\left\{\omega \in \Omega_{T}(M): \omega\left(t_{1}\right) \in A_{1}, \ldots, \omega\left(t_{n}\right) \in A_{n}\right\} \\
& =\int_{A_{1}} \cdots \int_{A_{n}} p\left(\delta_{0}, x_{0}, x_{1}\right) \cdots p\left(\delta_{n-1}, x_{n-1}, x_{n}\right) d \mu\left(x_{1}\right) \cdots d \mu\left(x_{n}\right)
\end{aligned}
$$

for all $n \in \mathbb{N}_{\geq 1}$, all partitions $0=t_{0}<t_{1}<\cdots<t_{n-1}<t_{n}=T$ and all Borel sets $A_{1}, \ldots, A_{n} \subset M$, where $\delta_{j}:=t_{j+1}-t_{j}$.

2. For every $x_{0}, y_{0} \in M$, the pinned Wiener measure $\mathbb{P}^{x_{0}, y_{0}, T}$ from $x_{0}$ to $y_{0}$ with terminal time $T$ is defined to be the unique probability measure on $\left(\Omega_{T}(M), \mathscr{F}^{T}\right)$ which satisfies

$$
\begin{aligned}
& \mathbb{P}^{x_{0}, y_{0}, T}(A)=\frac{1}{p\left(T, x_{0}, y_{0}\right)} \int_{A} p\left(T-t, \omega(t), y_{0}\right) d \mathbb{P}^{x_{0}, T}(\omega) \\
& \text { for all } 0 \leq t<T, \text { and all } A \in \mathscr{F}_{t}^{T} .
\end{aligned}
$$

It has been shown by Hsu (1990) that the pinned Wiener measure satisfies a natural large deviation principle under geodesic completeness.

The following well-known facts follow straightforwardly from the definitions and will be used repeatedly in the sequel:

Remark 2.2. 1. For every $x, y \in M$ one has

$$
\mathbb{P}^{x, T}\left\{\omega \in \Omega_{T}(M): \omega(0)=x\right\}=1=\mathbb{P}^{x, y, T}\left\{\omega \in \Omega_{T}(M): \omega(0)=x, \omega(T)=y\right\},
$$


as it should be. Furthermore $\mathbb{P}^{x, T}\left(\Omega_{T}(M)\right) \leq 1$, whereas $\mathbb{P}^{x, y, T}\left(\Omega_{T}(M)\right)=1$, which reflects the fact that "paths with explosion time" that are initially and terminally pinned on $M$ cannot explode.

2. One has the following time reversal symmetry of the pinned Wiener measure: The pushforward of $\mathbb{P}^{x, y, T}$ with respect to the $\mathscr{F}^{T} / \mathscr{F}^{T}$ measurable map $\Omega_{T}(M) \rightarrow$ $\Omega_{T}(M)$ given by $\omega \mapsto \omega(T-\cdot)$ is precisely $\mathbb{P}^{y, x, T}$.

3. For all $\mathscr{F}^{T}$-measurable functions $\Psi: \Omega_{T}(M) \rightarrow[0, \infty)$ one has Bär and Pfäffle (2011) the disintegration formula

$$
\int_{\Omega_{T}} \Psi(\omega) d \mathbb{P}^{x, T}(\omega)=\int_{M} p(T, x, y) \int_{\Omega_{T}} \Psi(\omega) d \mathbb{P}^{x, y, T}(\omega) d \mu(y) .
$$

4. Both measures $\mathbb{P}^{x, T}$ and $\mathbb{P}^{x, y, T}$ are concentrated on the set of $\alpha$-Hölder continuous paths $[0, T] \rightarrow M$, for every $\alpha \in(0,1 / 2)$ (cf. Bär and Pfäffle, 2011).

5 . We would also like to point out a technical issue, which is sometimes not taken into account seriously in the literature: assume $\left(\overline{\mathbb{P}^{x, T}}, \overline{\mathscr{F}^{T}}, \overline{\mathscr{F}_{*}^{T}}\right)$ denotes the minimal extension of $\left(\mathbb{P}^{x, T}, \mathscr{F}^{T}, \mathscr{F}_{*}^{T}\right)$ which satisfies the usual assumptions of rightcontinuity and completeness. Then there cannot exist a probability measure $Q$ on $\overline{\mathscr{F}}^{T}$ such that

$Q(A)=\frac{1}{p(T, x, y)} \int_{A} p(T-t, \omega(t), y) d \overline{\mathbb{P}^{x, T}}(\omega)$ for all $0 \leq t<T$, and all $A \in \overline{\mathscr{F}_{t}^{T}}$.

Namely, the existence of such a $Q$ would automatically imply the absolute continuity of $Q$ with respect to $\overline{\mathbb{P} x, T}$ on $\overline{\mathscr{F} T}$ (cf. Satz 5.40 in Hackenbroch and Thalmaier, 1994), which obviously cannot be true in view of

$$
Q\left\{\omega \in \Omega_{T}(M): \omega(T)=y\right\}=1 .
$$

If one needs some completeness of the underlying filtered probability space anyway (for example to get continuous versions of stochastic integrals) in addition to a relation of the form (2.1), one way out of this is to use the concept of "locally complete probability spaces", which to the best of our knowledge has been introduced in section 5.6 of Hackenbroch and Thalmaier (1994). Another possible solution is to complete the rough filtration with respect to the sum $\left(\mathbb{P}^{x, y, T}+\mathbb{P}^{x, T}\right) / 2$ (Driver, 1994). Nevertheless, as will be shown later on, the completeness of the underlying filtered probability space will play no role for the semimartingale property of the Brownian bridge.

Now we can give:

Definition 2.3. Let $(\Omega, \mathscr{F}, \mathbb{P})$ be a probability space, and let

$$
X^{x, y, T}:[0, T] \times \Omega \longrightarrow M
$$

be a continuous process ${ }^{1}$. Then $X^{x, y, T}$ is called a Brownian bridge from $x$ to $y$ with terminal time $T$ on $M$, if $\left(\widetilde{X^{x, y, T}}\right)_{*} \mathbb{P}=\mathbb{P}^{x, y, T}$, where

$$
\widetilde{X^{x, y, T}}: \Omega \longrightarrow \Omega_{T}(M), \widetilde{X^{x, y, T}}(\omega):=X^{x, y, T}(\omega)
$$

\footnotetext{
$1_{\text {that is, } X^{x, y, T}}(\omega)$ is continuous for all $\omega \in \Omega$, and $X_{t}^{x, y, T}$ is measurable for all $t \in[0, T]$, so that such a process automatically becomes jointly measurable; in the sequel we will identify indistinguishable processes.
} 
denotes the induced $\mathscr{F} / \mathscr{F}^{T}$ measurable map.

In this situation, if $X^{x, y, T}$ is adapted to a filtration $\mathscr{F}_{*}:=\left(\mathscr{F}_{t}\right)_{t \in[0, T]}$ of $\mathscr{F}$, then $X^{x, y, T}$ is called an $\mathscr{F}_{*}$-(adapted) Brownian bridge, if there holds the following timeinhomogeneous Markov property: for all numbers $0 \leq S<T$, all bounded $\mathscr{F}_{S^{-}}$ measurable $\Phi: \Omega \rightarrow \mathbb{R}$, and all bounded continuous (thus $\mathscr{F}^{T}$-measurable) $\Psi$ : $\Omega_{T}(M) \rightarrow \mathbb{R}$ one has

$$
\mathbb{E}\left[\Phi \cdot \Psi\left(X_{\min (S+\cdot, T)}^{x, y}\right)\right]=\mathbb{E}\left[\Phi \cdot \int \Psi\left(\omega(\min (\cdot, T-S)) d \mathbb{P}^{X_{S}^{x, y, T}, y, T-S}(\omega)\right]\right.
$$

We have defined the Brownian bridge in terms of its law. Instead, we could have defined the Brownian bridge as a diffusion having a time-dependent generator (cf. formula (1.3)). The latter definition leads to the problem that one has to deal right-away with the singularity of its generator at $T$, making even the continuity of the Brownian bridge paths at $T$ somewhat nonobvious. This is why we believe our definition in terms of the law is a little more natural in the bridge case. The price of our definition, on the other hand, is that it makes the proof of the semimartingale property in Theorem 2.8 below more complicated, as we have to calculate the generator by hand. Although the particular form of the generator is certainly wellknown to the experts, we have included a proof for the sake of completeness. This is essentially the content of Step 1 in the proof of Theorem 2.8 below.

The particular form of the Markov property from Definition 2.3 is motivated by the fact that every Brownian bridge satisfies this Markov property with respect to its own filtration:

Lemma 2.4. Let $(\Omega, \mathscr{F}, \mathbb{P})$ be a probability space, and let

$$
X^{x, y, T}:[0, T] \times \Omega \longrightarrow M
$$

be a Brownian bridge from $x$ to $y$ with terminal time $T$. Then, with $\mathscr{F}_{*}^{X^{x, y, T}}$ the filtration generated by $X^{x, y, T}$, one has the following time-inhomogeneous Markov property: For all numbers $0 \leq S<T$, all bounded $\mathscr{F}_{S}^{X^{x, y, T}}$-measurable $\Phi: \Omega \rightarrow \mathbb{R}$, and all bounded continuous (thus $\mathscr{F}^{T}$-measurable) $\Psi: \Omega_{T}(M) \rightarrow \mathbb{R}$ one has

$$
\mathbb{E}\left[\Phi \cdot \Psi\left(X_{\min (S+\cdot, T)}^{x, y, T}\right)\right]=\mathbb{E}\left[\Phi \cdot \int \Psi(\omega(\min (\cdot, T-S))) d \mathbb{P}_{S}^{X_{S}^{x, y, T}, y, T-S}(\omega)\right],
$$

in other words, $X^{x, y, T}$ is an $\mathscr{F}_{*}^{X^{x, y, T}}$-Brownian bridge.

Proof: As by the Doob-Dynkin lemma (cf. proof of Lemma V.15 in Güneysu, 2017) one can write $\Phi=F\left(X^{x, y, T}\right)$ for some bounded $\mathscr{F}_{S}^{T}$-measurable function $F$ : $\Omega_{T}(M) \rightarrow \mathbb{R}$, we can and we will assume that the underlying filtered probability space is given by $\left(\Omega_{T}(M), \mathscr{F}^{T}, \mathscr{F}_{*}^{T}, \mathbb{P}^{x, y, T}\right)$ with its coordinate process $X$. After this observation, we can follow the proof of the Euclidean case which is given in the book Sznitman (1998), pp. 139/140: For all $0<\delta<T$ we have, with $X$ the 
coordinate process on $\Omega_{T}(M)$,

$$
\begin{aligned}
& p(T, x, y) \mathbb{E}^{x, y, T}\left[\Phi \cdot \int \Psi(\omega(\min (\cdot, T-S-\delta))) d \mathbb{P}^{X_{S}^{x, y, T}, y, T-S}(\omega)\right] \\
& =\mathbb{E}^{x, T}\left[\Phi \cdot p\left(T-S, X_{S}, y\right) \int \Psi(\omega(\min (\cdot, T-S-\delta))) d \mathbb{P}^{X_{S}^{x, y, T}, y, T-S}(\omega)\right] \\
& =\mathbb{E}^{x, T}\left[\Phi \cdot \int \Psi(\omega(\min (\cdot, T-S-\delta))) p(\delta, \omega(T-\delta-S), y) d \mathbb{P}_{S}^{X_{S}^{x, y, T}, T-S}(\omega)\right] \\
& =\mathbb{E}^{x, T}\left[\Phi \cdot \Psi\left(X_{\min (S+\cdot, T-\delta)}\right) p\left(\delta, X_{T-\delta}, y\right)\right] \\
& =p(T, x, y) \mathbb{E}^{x, y, T}\left[\Phi \cdot \Psi\left(X_{\min (S+\cdot, T-\delta)}\right)\right],
\end{aligned}
$$

where we have used the defining relation of the pinned Wiener measure for the first two equalities, the usual Markov property of the Wiener measure (in the sense of a sub-probability measure) for the third equality, and once again the defining relation of the pinned Wiener measure for the last equality. Finally, taking $\delta \rightarrow 0+$ completes the proof using dominated convergence, noting that $\Psi$ is continuous and bounded.

Remark 2.5. 1. As a consequence of Lemma 2.4 we get that the coordinate process

$$
X:[0, T] \times \Omega \longrightarrow M, \quad X_{t}(\omega)=\omega(t) \quad \text { on } \quad\left(\Omega_{T}, \mathscr{F}^{T}, \mathscr{F}_{*}^{T}, \mathbb{P}^{x, y, T}\right)
$$

is an $\mathscr{F}_{*}^{T}$-adapted Brownian bridge.

2. Another possible construction (Hsu, 2002) of an adapted Brownian bridge is provided by adopting the construction of Brownian motion on $M$ by projecting the solution of the canonical stochastic differential equation on the orthonormal frame bundle $O(M)$ of $M$ (which is given by the horizontal vector fields on $O(M)$ and a standard Euclidean Brownian motion). In the bridge case, one has to add a drift term which contains a singularity near $T$, so that a priori one only gets a unique solution which is defined on the time interval $[0, T)$ and it becomes a somewhat technical issue to prove that this solution has a limit at $T$ and indeed is a Brownian bridge.

3. A third approach (Bismut, 1984b), which like the path space approach has the advantage of avoiding the singularity at $T$, is as follows: one starts from a Brownian motion $X^{x}$ on $M$ which is obtained on the $m$-dimensional standard Euclidean Wiener space $\left(\Omega, \mathscr{F}, \mathscr{F}_{*}, \mathbb{P}\right)$ by projecting the solution of the canonical stochastic differential equation on the orthonormal frame bundle $O(M)$. Here one assumes that $\left(\Omega, \mathscr{F}, \mathscr{F}_{*}, \mathbb{P}\right)$ satisfies the usual assumptions. Then one would like to define a probability measure $P^{x, y, T}$ on $\mathscr{F}_{T}$ such that for all $t<T, A \in \mathscr{F}_{t}$, one has

$$
P^{x, y, T}(A)=\frac{1}{p(T, x, y)} \int_{A} p\left(T-t, X_{t}^{x}, y\right) d \mathbb{P} .
$$

Then, $\left.X(x)\right|_{[0, T]}$ would indeed be an adapted Brownian bridge

$$
\text { on }\left(\Omega, \mathscr{F}_{T},\left.\mathscr{F}_{*}\right|_{[0, T]}, P^{x, y, T}\right) .
$$

However, again due to Satz 5.40 in Hackenbroch and Thalmaier (1994) such a measure cannot exist, and again the way out of this is to start with the 'rough' (that is, not yet completed) $m$-dimensional standard Euclidean Wiener space and to carry out the above construction on its local completion. 
As we allow filtered probability spaces with a finite time horizon that need not satisfy the usual assumptions, a somewhat nonstandard setting for semimartingales on manifolds, we add:

Remark 2.6. In the sequel, given $T>0$ and a filtered probability space $\left(\Omega, \mathscr{F},\left(\mathscr{F}_{t}\right)_{t \in[0, T]}, \mathbb{P}\right)$ we say that an increasing sequence $T_{n}: \Omega \rightarrow[0, T], n \in \mathbb{N}$, of $\mathscr{F}_{*}$-stopping times announces $T$, if

$$
\lim _{n \rightarrow \infty} \mathbb{P}\left(T_{n}=T\right)=1 .
$$

As usual, we will say that a process $Y$ which is defined on $[0, T] \times \Omega$ has $\mathscr{F}_{*}$-locally a certain property $\mathscr{P}$, if for some sequence $T_{n}: \Omega \rightarrow[0, T], n \in \mathbb{N}$ of $\mathscr{F}_{*}$-stopping times which announces $T$, the process $Y_{\min \left(\cdot, T_{n}\right)}$ has the property $\mathscr{P}$. A process

$$
Y:[0, T] \times \Omega \longrightarrow \mathbb{R}
$$

is called a continuous semimartingale w.r.t. $\mathscr{F}_{*}:=\left(\mathscr{F}_{t}\right)_{t \in[0, T]}$, if there exist continuous processes

$$
Y^{(1)}:[0, T] \times \Omega \longrightarrow \mathbb{R}, \quad Y^{(2)}:[0, T] \times \Omega \longrightarrow \mathbb{R}
$$

such that

- $Y^{(1)}$ is adapted to $\mathscr{F}_{*}$ with paths $\mathscr{F}_{*}$-locally having a finite variation

- $Y^{(2)}$ is $\mathscr{F}_{*}$-locally a martingale starting from 0

- $Y=Y^{(1)}+Y^{(2)}$.

We recall further that, following Laurent Schwartz, a continuous $\mathscr{F}_{*}$-adapted manifold-valued process

$$
X:[0, T] \times \Omega \longrightarrow M
$$

is called a continuous semimartingale w.r.t. $\mathscr{F}_{*}$, if for all smooth $f: M \rightarrow \mathbb{R}$ the process

$$
f(X):[0, T] \times \Omega \longrightarrow \mathbb{R}
$$

is a real-valued continuous $\mathscr{F}_{*}$-semimartingale in the sense of the former definition. With this definition, it follows that if

$$
X:[0, T] \times \Omega \longrightarrow M
$$

is a continuous semimartingale w.r.t. $\left(\Omega, \mathscr{F},\left(\mathscr{F}_{t}\right)_{t \in[0, T]}, \mathbb{P}\right)$, then it is also one w.r.t. the minimal extension of the latter filtration which satisfies the usual assumptions (of completeness and right-continuity). Furthermore, a process

$$
X:[0, T] \times \Omega \longrightarrow M
$$

is a continuous $\mathscr{F}_{*}$-semimartingale, if and only if it is $\mathscr{F}_{*}$-locally a continuous $\mathscr{F}_{*}$ semimartingale.

The latter probabilistic localization leads to a simple geometric localization:

Lemma 2.7. Assume we are given $T>0$, a filtered probability space $\left(\Omega, \mathscr{F},\left(\mathscr{F}_{t}\right)_{t \in[0, T]}, \mathbb{P}\right)$, and a continuous $\mathscr{F}_{*}:=\left(\mathscr{F}_{t}\right)_{t \in[0, T]}$-adapted continuous process

$$
X:[0, T] \times \Omega \longrightarrow M
$$

such that for all smooth compactly supported $\phi: M \rightarrow \mathbb{R}$ the process $\phi(X)$ is a continuous $\mathscr{F}_{*}$-semimartingale. Then $X$ is a continuous $\mathscr{F}_{*}$-semimartingale. 
Proof: Let $f$ be an arbitrary smooth function on $M$, let $\left(U_{n}\right)_{n \in \mathbb{N}}$ be an open relatively compact exhaustion of $M$. Then, as $X$ is continuous and adapted, and $U_{n}$ is open, the first exit time $T_{n}: \Omega \rightarrow[0, T]$,

$$
T_{n}:=\left\{\begin{array}{l}
\inf \left\{t \in[0, T]: X_{t} \in M \backslash U_{n}\right\}, \quad \text { if }\{\ldots\} \neq \emptyset \\
T, \text { else }
\end{array},\right.
$$

of $X$ from $U_{n}$ is a stopping time, for each $n \in \mathbb{N}$, and $\left(T_{n}\right)_{n \in \mathbb{N}}$ announces $T$. If $\phi_{n}$ is a smooth compactly supported function $M$ with $\phi_{n} \equiv 1$ on $\overline{U_{n}}$, then by assumption $\left(\phi_{n} f\right)(X)$ is a continuous semimartingale which coincides with $f\left(X_{\min \left(\cdot, T_{n}\right)}\right)$.

In the sequel, for every fixed $y \in M$ we define the strictly positive space-time function

$$
p^{y}:(0, \infty) \times M \longrightarrow(0, \infty), p^{y}(t, x):=p(t, x, y) .
$$

Now we can formulate our main result:

Theorem 2.8. Let $M$ be geodesically complete, let $x, y \in M, T>0$, let $\left(\Omega, \mathscr{F},\left(\mathscr{F}_{t}\right)_{t \in[0, T]}, \mathbb{P}\right)$ be a filtered probability space, and assume furthermore that $X^{x, y, T}$ is an $\mathscr{F}_{*}:=\left(\mathscr{F}_{t}\right)_{t \in[0, T]}$-Brownian bridge from $x$ to $y$ with terminal time $T$. Then for all smooth compactly supported $f: M \rightarrow \mathbb{R}$ one has

$$
\mathbb{E}\left[\int_{0}^{T}\left|d \log p^{y}\left(T-r, X_{r}^{x, y, T}\right)\right|\left|d f\left(X_{r}^{x, y, T}\right)\right| d r\right]<\infty,
$$

and the real-valued process

$$
\begin{aligned}
& X^{x, y, T, f}:[0, T] \times \Omega \longrightarrow \mathbb{R} \\
& X_{s}^{x, y, T, f}:= f\left(X_{s}^{x, y, T}\right)-f\left(X_{0}^{x, y, T}\right)-(1 / 2) \int_{0}^{s} \Delta f\left(X_{r}^{x, y, T}\right) d r \\
&\left.\quad-\int_{0}^{s}\left(d \log p^{y}\left(T-r, X_{r}^{x, y, T}\right), d f\left(X_{r}^{x, y, T}\right)\right)\right) d r
\end{aligned}
$$

is a continuous $\mathscr{F}_{*}$-martingale. In particular, $X^{x, y, T}$ is a continuous semimartingale with respect to $\mathscr{F}_{*}$.

The following localized $C^{1}$-heat kernel bounds will play a central role in the proof of Theorem 2.8:

Theorem 2.9. Let $M$ be geodesically complete.

a) For every $z_{0} \in M$ and every $R>0$ there exist constants $C_{j}>0$ (which depend on the geometry of $M$ in a neighbourhood of $\left.B\left(z_{0}, R\right)\right)$ such that for all $(t, x, y) \in$ $(0, R] \times B\left(z_{0}, R\right) \times B\left(z_{0}, R\right)$ one has

$$
C_{1} t^{-m / 2} e^{-C_{2} \frac{d(x, y)^{2}}{t}} \leq p(t, x, y) \leq C_{3} t^{-m / 2} e^{-C_{4} \frac{d(x, y)^{2}}{t}} .
$$

b) For every $z_{0} \in M$ and every $R>0$ there exists a constant $C>0$ (which depends on the geometry of $M$ in a neighbourhood of $B\left(z_{0}, R\right)$ ) such that for all $(t, x, y) \in\left(0, R^{2}\right] \times B\left(z_{0}, R\right) \times B\left(z_{0}, R\right)$ one has

$$
\left|d \log p^{y}(t, x)\right| \leq C\left(t^{-1 / 2}+t^{-1} d(x, y)\right),
$$

where here and in the sequel $d \log p^{y}(t, x):=d_{x} \log p^{y}(t, x)$, that is, the exterior differential of a function on space-time is always understood with respect to the space variable. 
While the proof of Theorem 2.9 a) is consequence of localized versions of standard comparison results in Riemannian geometry (cf. appendix A), the proof of Theorem $2.9 \mathrm{~b}$ ) is based on the following localized version of Hamilton's gradient estimate, which is by Arnaudon and Thalmaier:

Theorem 2.10. Let $D \subset M$ be an open relatively compact subset, let $S>0$, and let a continuous function

$$
u:[0, S] \times \bar{D} \longrightarrow(0, \infty)
$$

be given, which is a smooth solution of

$$
\frac{\partial}{\partial s} u(s, z)=\frac{1}{2} \Delta u(s, z) \quad \text { in }(s, z) \in[0, S] \times D .
$$

Then for all $K \geq 0$ with $\left.\mathrm{Ric}\right|_{D} \geq-K$, all $\beta>0, w \in D$, and all $z \in$ $B(w, d(w, \partial D) / 2)$ one has the gradient bound

$$
|d \log u(z, S)|^{2} \leq 2\left(\frac{1}{S}+\frac{\pi^{2}(m+\beta m+7)}{d(w, \partial D)^{2}}+\frac{K}{4 \beta}+K\right)\left(4+\log \frac{\sup _{[0, S] \times \bar{D}} u}{u(z, S)}\right)^{2} .
$$

Theorem 2.10 is an immediate consequence of Theorem 7.1 in Arnaudon and Thalmaier (2010), where an entirely probabilistic proof has been given. We refer the reader also to Thalmaier and Wang (1998) for analogous techniques.

Proof of Theorem 2.9: a) The localized heat kernel bounds that we have recorded in the appendix (cf. appendix A), show the existence of constants $A_{j}>0, j=1, \ldots, 4$, that only depend on $m$ and a lower bound of Ric in a neighbourhood of $B\left(z_{0}, R\right)$, such that for all $(t, x, y) \in\left(0, R^{2}\right] \times B\left(z_{0}, R\right) \times B\left(z_{0}, R\right)$ one has

$$
\begin{aligned}
& e^{-A_{1} t} \mu\left(B(x, \sqrt{t})^{-1 / 2} \mu(B(y, \sqrt{t}))^{-1 / 2} e^{-A_{2} \frac{d(x, y)^{2}}{t}}\right. \\
& \leq p(t, x, y) \\
& \leq e^{A_{3} t} \mu(B(x, \sqrt{t}))^{-1 / 2} \mu\left(B(y, \sqrt{t})^{-1 / 2} e^{-A_{4} \frac{d(x, y)^{2}}{t}} .\right.
\end{aligned}
$$

As $\sqrt{t} \leq R<R+1$ and $B(x, 2 \sqrt{t}) \subset B\left(z_{0}, 4(R+1)\right), B(y, 2 \sqrt{t}) \subset B\left(z_{0}, 4(R+1)\right)$, applying Bishop-Gromov's volume estimates locally (cf. appendix A) we can pick $A_{5}, A_{6}>0$ (that only depend on $m$ and a lower bound of Ric on say $B\left(z_{0}, 4(R+1)\right)$ ), such that

$$
\max \{\mu(B(x, \sqrt{t})), \mu(B(y, \sqrt{t}))\} \leq A_{5} t^{m / 2} e^{A_{6} \sqrt{t}} \leq A_{5} e^{A_{6} \sqrt{R}} t^{m / 2},
$$

which yields the estimate

$$
p(t, x, y) \geq A_{5} e^{-A_{1} R} e^{-A_{6} \sqrt{R}} t^{-m / 2} e^{-A_{2} \frac{d(x, y)^{2}}{t}} .
$$

On the other hand, using again $\sqrt{t} \leq R<R+1, B(x, 2 \sqrt{t}) \subset B\left(z_{0}, 4(R+1)\right)$, $B(y, 2 \sqrt{t}) \subset B\left(z_{0}, 4(R+1)\right)$, and applying a local volume doubling inequality (cf. appendix A), we can pick $A_{7}>0$ (that only depends on $m$ and a lower bound of Ric on $\left.B\left(z_{0}, 4(R+1)\right)\right)$ such that

$$
\begin{aligned}
& \min \{\mu(B(x, \sqrt{t})), \mu(B(y, \sqrt{t}))\} \\
& \geq(R+1)^{-m} e^{-A_{7}(R+1)} \inf _{a \in B\left(z_{0}, R\right)} \mu(B(a, R+1)) t^{-m / 2} \\
& =: A_{8} t^{-m / 2} .
\end{aligned}
$$


Thus we have

$$
p(t, x, y) \leq e^{A_{3} R} A_{8} t^{-m / 2} e^{-A_{4} \frac{d(x, y)^{2}}{t}},
$$

completing the proof.

b) We will use Theorem 2.10 as follows: Define $S:=t / 2, u(s, z):=p^{y}(s+t / 2, z)$ and let $D:=B\left(z_{0}, 2 R\right)$. We can pick finitely many $w_{1}, \ldots w_{l} \in D$ such that $B\left(z_{0}, R\right) \subset \bigcup_{j=1}^{l} B\left(w_{j}, d\left(w_{j}, \partial D\right) / 2\right)$. Then with the above choices Theorem 2.10 immediately implies

$$
\begin{aligned}
\left|d \log p^{y}(x, t)\right|^{2} & \leq 2\left(\frac{2}{t}+\frac{\pi^{2}(m+\beta m+7)}{\min _{j=1, \ldots, l} d\left(w_{j}, \partial D\right)^{2}}+\frac{K}{4 \beta}+K\right) \\
& \times\left(4+\log \frac{\sup _{s \in[0, t / 2], z \in \bar{D}} p(t / 2+s, z, y)}{p(t, x, y)}\right)^{2},
\end{aligned}
$$

where $K \geq 0$ is chosen such that $-K$ is a lower bound on the Ricci curvature on $D=B\left(z_{0}, 2 R\right)$, and $\beta>0$ can be chosen arbitrarily. Finally, by part a), we can find constants $c_{j}>0$ such that

$$
\log \frac{\sup _{s \in[0, t / 2], z \in \bar{D}} p(t / 2+s, z, y)}{p(x, y, t)} \leq c_{1}+c_{2} d(x, y)^{2} / t,
$$

showing the inequality

$$
\left|d \log p^{y}(t, x)\right| \leq C\left(t^{-1 / 2}+t^{-1} d(x, y)+t^{-1 / 2} d(x, y)+1\right),
$$

which proves the claim (noting that $1 \leq R^{2} / t$ and $t^{-1 / 2} \leq R t^{-1}$.

Proof of Theorem 2.8: Let $R_{0}>0, z_{0} \in M$ be arbitrary. We prove the claim for $x, y \in B\left(z_{0}, R_{0}\right)$. To this end, we fix an arbitrary smooth compactly supported $f: M \rightarrow \mathbb{R}$.

The proof is divided into four parts:

Claim 1: With $p^{y}:=p(\cdot, \cdot, y)$, for every $0 \leq t<s<T$, and $A \in \mathscr{F}_{t}$ one has

$$
\begin{aligned}
& \frac{d}{d s} \mathbb{E}\left[1_{A} f\left(X_{s}^{x, y, T}\right)\right] \\
& =\mathbb{E}\left[1_{A}\left((1 / 2) \Delta f\left(X_{s}^{x, y, T}\right)+\left(d \log p^{y}\left(T-s, X_{s}^{x, y, T}\right), d f\left(X_{s}^{x, y, T}\right)\right)\right)\right] .
\end{aligned}
$$

Proof of Claim 1: In principle we follow the compact case Driver (1994) here, up to the fact that we have to use the Markov property of the bridge (which makes the calculation more complicated): Using the time-inhomogeneous Markov property of $X^{x, y, T}$ and the defining relation of the pinned Wiener measure (note that $s-t<T-t$ ), we can calculate

$$
\begin{aligned}
& \mathbb{E}\left[1_{A} f\left(X_{s}^{x, y, T}\right)\right]=\mathbb{E}\left[1_{A} \int f(\omega(s-t)) d \mathbb{P}^{X_{t}^{x, y, T}, y, T-t}(\omega)\right] \\
& =\mathbb{E}\left[1_{A} \frac{1}{p^{y}\left(T-t, X_{t}^{x, y, T}\right)} \int p^{y}(T-s, \omega(s-t)) f(\omega(s-t)) d \mathbb{P}^{X_{t}^{x, y, T}, T-t}(\omega)\right] .
\end{aligned}
$$

Let us define a smooth function

$$
\Psi:[0, T) \times M \longrightarrow \mathbb{R}, \Psi_{r}(z):=p^{y}(T-r, z) f(z),(r, z) \in[0, T) \times M .
$$


Then, using that the heat kernel solves the heat equation and that $\Delta$ is formally self-adjoint (note here that $\Psi_{s}$ has a compact support in $M$ ), one can easily deduce

$$
\frac{\partial}{\partial s} P_{s-t} \Psi_{s}(z)=P_{s-t}\left[\left(\frac{\partial}{\partial s}+(1 / 2) \Delta\right) \Psi_{s}\right](z),
$$

an expression, which using the product rule for the Laplace-Beltrami operator and once more that the heat kernel solves the heat equation, is seen to be equal to

$$
\begin{aligned}
& =P_{s-t}\left[p^{y}(T-s, \cdot)(1 / 2) \Delta f+\left(d p^{y}(T-s, \cdot), d f\right)\right](z) \\
& =P_{s-t}\left[p^{y}(T-s, \cdot)(1 / 2) \Delta f+p^{y}(T-s, \cdot)\left(d \log p^{y}(T-s, \cdot), d f\right)\right](z) .
\end{aligned}
$$

Thus, using (2.2) and using the defining relation of the Wiener measure twice,

$$
\begin{aligned}
& \frac{d}{d s} \mathbb{E}\left[1_{A} f\left(X_{s}^{x, y, T}\right)\right]=\frac{d}{d s} \mathbb{E}\left[1_{A} \frac{1}{p^{y}\left(T-t, X_{t}^{x, y, T}\right)} P_{s-t} \Psi_{s}\left(X_{t}^{x, y, T}\right)\right] \\
& =\mathbb{E}\left[1_{A} \frac{1}{p^{y}\left(T-t, X_{t}^{x, y, T}\right)} \frac{\partial}{\partial s} P_{s-t} \Psi_{s}\left(X_{t}^{x, y, T}\right)\right] \\
& =\mathbb{E}\left[1_{A} \frac{1}{p^{y}\left(T-t, X_{t}^{x, y, T}\right)}\right. \\
& \left.\quad \times P_{s-t}\left[p^{y}(T-s, \cdot)(1 / 2) \Delta f+p^{y}(T-s, \cdot)\left(d \log p^{y}(T-s, \cdot), d f\right)\right]\left(X_{t}^{x, y, T}\right)\right] \\
& =\mathbb{E}\left[1_{A} \frac{1}{p^{y}\left(T-t, X_{t}^{x, y, T}\right)} \int p^{y}(T-s, \omega(s-t))\right. \\
& \left.\times\left((1 / 2) \Delta f(\omega(s-t))+\left(d \log p^{y}(T-s, \omega(s-t)), d f(\omega(s-t))\right)\right) d \mathbb{P}^{X_{t}^{x, y, T}, T-t}(\omega)\right] .
\end{aligned}
$$

In view of the defining relation of the pinned Wiener measure, we see that the latter expression is

$$
\begin{aligned}
=\mathbb{E} & {\left[1_{A} \int\left((1 / 2) \Delta f(\omega(s-t))+\left(d \log p^{y}(T-s, \omega(s-t)), d f(\omega(s-t))\right)\right)\right.} \\
& \left.\times d \mathbb{P}^{X_{t}^{x, y, T}, y, T-t}(\omega)\right] .
\end{aligned}
$$

That we can indeed interchange $\frac{d}{d s}$ and $\mathbb{E}$ in (2.3) is seen as follows: Fix arbitrary $t, T, \epsilon>0$ with $0<t<T-\epsilon$. Then $\mathbb{P}$-a.s., for all $s$ with $t<s<T-\epsilon$ the above calculation inside $\mathbb{E}[\cdots]$ shows

$$
\begin{aligned}
& \left|\frac{\partial}{\partial s} 1_{A} f\left(X_{s}^{x, y, T}\right)\right| \\
& \leq(1 / 2) \int|\Delta f(\omega(s-t))| d \mathbb{P}^{X_{t}^{x, y, T}, y, T-t}(\omega) \\
& \quad+\int\left|\left(d \log p^{y}(T-s, \omega(s-t)), d f(\omega(s-t))\right)\right| d \mathbb{P}^{X_{t}^{x, y, T}, y, T-t}(\omega) \\
& \leq(1 / 2)\|\Delta f\|_{\infty}+\|d f\|_{\infty} \sup _{s \in[t, T-\epsilon), z \in \operatorname{supp}(f)}\left|d \log p^{y}(T-s, z)\right| .
\end{aligned}
$$


Finally, using the time-inhomogeneous Markov property of $X^{x, y, T}$ we get that (2.4) implies

$$
\begin{aligned}
& \frac{d}{d s} \mathbb{E}\left[1_{A} f\left(X_{s}^{x, y, T}\right)\right] \\
& =\mathbb{E}\left[1_{A}\left((1 / 2) \Delta f\left(X_{s}^{x, y, T}\right)+\left(d \log p^{y}\left(T-s, X_{s}^{x, y, T}\right), d f\left(X_{s}^{x, y, T}\right)\right)\right)\right],
\end{aligned}
$$

which completes the proof of Claim 1 .

Claim 2: One has

$$
\mathbb{E}\left[\int_{0}^{T}\left|d \log p^{y}\left(T-r, X_{r}^{x, y, T}\right)\right|\left|d f\left(X_{r}^{x, y, T}\right)\right| d r\right]<\infty .
$$

Proof of Claim 2: Using the time reversal symmetry of the pinned Wiener measure we have (with $X$ the coordinate process on $\Omega_{T}(M)$ )

$$
\begin{aligned}
& \mathbb{E}^{x, y, T}\left[\int_{0}^{T}\left|d \log p^{y}\left(T-r, X_{r}\right)\right|\left|d f\left(X_{r}\right)\right| d r\right] \\
& =\mathbb{E}^{x, y, T}\left[\int_{0}^{T / 2}\left|d \log p^{y}\left(T-r, X_{r}\right)\right|\left|d f\left(X_{r}\right)\right| d r\right] \\
& +\mathbb{E}^{y, x, T}\left[\int_{0}^{T / 2}\left|d \log p^{y}\left(r, X_{r}\right)\right|\left|d f\left(X_{r}\right)\right| d r\right] .
\end{aligned}
$$

For the first summand we have

$$
\mathbb{E}^{x, y, T}\left[\left|d \log p^{y}\left(T-r, X_{r}\right)\right|\left|d f\left(X_{r}\right)\right|\right] \leq \sup _{z \in \operatorname{supp}(f), u \in[T / 2, T]}\left|d \log p^{y}(u, z)\right||d f(z)|,
$$

for every $0<r \leq T / 2$, as in this range we stay away from the heat kernel singularity. In particular, the first summand is finite.

It remains to estimate the second summand. To this end, we pick $R>\max \left(R_{0}, T^{2}\right)$ large enough such that $B\left(z_{0}, R\right) \supset \operatorname{supp}(f)$. Using again the defining relation of the pinned Wiener measure, and of the Wiener measure, respectively, we have for every $0<r \leq T / 2$,

$$
\begin{aligned}
& p(T, y, x) \mathbb{E}^{y, x, T}\left[\left|d \log p^{y}\left(r, X_{r}\right)\right|\left|d f\left(X_{r}\right)\right|\right] \\
& =\int p(r, y, z) p(T-r, z, x)\left|d \log p^{y}(r, z)\right||d f(z)| d \mu(z) \\
& \leq \int_{B\left(z_{0}, R\right)} p(r, y, z) p(T-r, z, x)\left|d \log p^{y}(r, z)\right||d f(z)| d \mu(z) \\
& \leq\|d f\|_{\infty} \int_{B\left(z_{0}, R\right)} p(r, y, z) p(T-r, z, x)\left|d \log p^{y}(r, z)\right| d \mu(z) \\
& \leq\left\{\sup _{u \in[T / 2, T], a, b \in B\left(z_{0}, R\right)} p(u, a, b)\right\}\|d f\|_{\infty} \int_{B\left(z_{0}, R\right)} p(r, y, z)\left|d \log p^{y}(r, z)\right| d \mu(z) \\
& =: A \int_{B\left(z_{0}, R\right)} p(r, y, z)\left|d \log p^{y}(r, z)\right| d \mu(z) .
\end{aligned}
$$

Next, using Theorem 2.9, we pick a constant $C>0$ such that for all $u \in(0, T]$, $x_{1}, x_{2} \in B\left(z_{0}, R\right)$ one has

$$
\left|d \log p\left(u, x_{1}, x_{2}\right)\right| \leq C u^{-1 / 2}+C u^{-1} d\left(x_{1}, x_{2}\right)
$$


and

$$
p\left(u, x_{1}, x_{2}\right) \leq C u^{-m / 2} e^{-C \frac{d\left(x_{1}, x_{2}\right)^{2}}{u}},
$$

so that using $\int_{M} p(\alpha, v, w) d \mu(w) \leq 1$ for all $\alpha>0, v \in M$,

$$
\begin{aligned}
& A^{-1} p(T, y, x) \mathbb{E}^{y, x, T}\left[\left|d \log p^{y}\left(r, X_{r}\right)\right|\left|d f\left(X_{r}\right)\right|\right] \\
& \leq C r^{-1 / 2} \int_{B\left(z_{0}, R\right)} p(r, y, z) d \mu(z)+C r^{-1} \int_{B\left(z_{0}, R\right)} p(r, y, z) d(y, z) d \mu(z) \\
& \leq C r^{-1 / 2}+C^{2} r^{-1-m / 2} \int_{B\left(z_{0}, R\right)} e^{-C \frac{d(z, y)^{2}}{r}} d(y, z) d \mu(z) .
\end{aligned}
$$

The first summand is integrable in $r$ from 0 to $T / 2$. For the second summand we proceed as follows: Pick $R^{\prime}$ large with $B\left(z_{0}, R\right) \subset B\left(y, R^{\prime}\right)$. Then using the co-area formula

$$
\begin{aligned}
& r^{-1-m / 2} \int_{B\left(z_{0}, R\right)} e^{-C \frac{d(z, y)^{2}}{r}} d(y, z) d \mu(z) \leq r^{-1-m / 2} \int_{B\left(y, R^{\prime}\right)} e^{-C \frac{d(z, y)^{2}}{r}} d(y, z) d \mu(z) \\
& =r^{-1-m / 2} \int_{0}^{R^{\prime}} e^{-C \frac{u^{2}}{r}} \cdot u \cdot \operatorname{area}(\{z \in M: d(z, y)=u\}) d u \\
& \leq c r^{-1-m / 2} \int_{0}^{R^{\prime}} e^{-C \frac{u^{2}}{r}} u u^{m-1} d u \leq c^{\prime} r^{-1-m / 2+m / 2+1 / 2}=c^{\prime} r^{-1 / 2},
\end{aligned}
$$

for some $c, c^{\prime}>0$, where we also have used the Bishop-Gromov estimate for metric spheres from appendix $A$, and where we have estimated the last integral with

$$
\int_{0}^{R^{\prime}} e^{-C \frac{u^{2}}{r}} u^{m} d u \leq \int_{0}^{\infty} e^{-C \frac{u^{2}}{r}} u^{m} d u=C_{m}(C / r)^{-m / 2-1 / 2}
$$

Again, the result is an integrable function of $r$ in $[0, T / 2]$. This completes the proof of Claim 2.

Claim 3: The real-valued process

$$
\begin{aligned}
Y:= & X^{x, y, T, f}:[0, T] \times \Omega \longrightarrow \mathbb{R} \\
Y_{s}:= & f\left(X_{s}^{x, y, T}\right)-f\left(X_{0}^{x, y, T}\right)-(1 / 2) \int_{0}^{s} \Delta f\left(X_{r}^{x, y, T}\right) d r \\
& \left.-\int_{0}^{s}\left(d \log p^{y}\left(T-r, X_{r}^{x, y, T}\right), d f\left(X_{r}^{x, y, T}\right)\right)\right) d r
\end{aligned}
$$

is a continuous $\mathscr{F}_{*}$-martingale.

Proof of Claim 3: It follows from applying $\int_{t}^{s} \cdots$ to the formula from Claim 1 that for all $0 \leq t<s \leq T$, all $A \in \mathscr{F}_{t}$, and all $n \in \mathbb{N}$ one has

$$
\mathbb{E}\left[1_{A}\left(Y_{t}-Y_{s-1 / n}\right)\right]=0 \text {. }
$$

As $f$ has a compact support, we can use Claim 2 and dominated convergence to deduce

$$
\mathbb{E}\left[1_{A}\left(Y_{t}-Y_{s}\right)\right]=0
$$

which is equivalent to

$$
\mathbb{E}\left[Y_{t} \mid \mathscr{F}_{s}\right]=Y_{s} \quad \mathbb{P} \text {-a.s. }
$$

Claim 4: $f\left(X^{x, y, T}\right)$ is a continuous $\mathscr{F}_{*}$ semimartingale. 
Proof of Claim 4: We have for all $s \in[0, T], \mathbb{P}$-a.s.

$$
\begin{aligned}
f\left(X_{s}^{x, y, T}\right)= & Y_{s}+f\left(X_{0}^{x, y, T}\right)+(1 / 2) \int_{0}^{s} \Delta f\left(X_{r}^{x, y, T}\right) d r \\
& \left.+\int_{0}^{s}\left(d \log p^{y}\left(T-r, X_{r}^{x, y, T}\right), d f\left(X_{r}^{x, y, T}\right)\right)\right) d r .
\end{aligned}
$$

Thus by Claim 2 and Claim 3 this is a sum of a continuous local martingale and a continuous adapted process with paths having a finite variation. This completes the proof.

Let now $G$ be a Lie-group with $\mathfrak{g}$ its Lie-algebra. By standard results on manifoldvalued continuous semimartingales (Hackenbroch and Thalmaier, 1994), we have:

Corollary 2.11. Let $\left(\Omega, \mathscr{F},\left(\mathscr{F}_{t}\right)_{t \in[0, T]}, \mathbb{P}\right)$ be a filtered probability space which satisfies the usual assumptions, and let $X^{x, y, T}$ be an $\mathscr{F}_{*}:=\left(\mathscr{F}_{t}\right)_{t \in T}$-Brownian bridge $X^{x, y, T}$ from $x$ to $y$ with terminal time $T$. Assume further that we are given a smooth principal $G$-bundle $\pi: P \rightarrow M$ together with a connection 1-form $\alpha \in \Omega_{C^{\infty}}^{1}(P, \mathfrak{g})$. Then for every $\mathscr{F}_{0}$-measurable random variable $u: \Omega \rightarrow P$ with $\pi(u)=x \mathbb{P}$-a.s., there exists a unique (up to indistinguishability) $\alpha$-horizontal $\mathscr{F}_{*}$ lift $U:[0, T] \times \Omega \rightarrow P$ of $X^{x, y, T}$ with $U_{0}=u \mathbb{P}$-a.s. In other words, $U$ is the uniquely determined continuous $\mathscr{F}_{*}$-semimartingale $U:[0, T] \times \Omega \rightarrow P$ which satisfies the following properties:

- $U_{0}=u \mathbb{P}$-a.s.

- $\pi\left(U_{t}\right)=X_{t}^{x, y, T} \mathbb{P}$-a.s., for all $t \in[0, T]$

- $\int_{0}^{t} \alpha\left(\underline{d} U_{s}\right)=0$ (Stratonovic line integral of $\alpha$ along $\left.U\right) \mathbb{P}$-a.s., for all $t \in$ $[0, T]$.

\section{Appendix A. Localized heat kernel and volume bounds}

For the convenience of the reader we record here some facts on heat-kernels and volumes on geodesically complete Riemannian manifolds. Let $M$ be a geodesically complete connected smooth Riemannian $m$-manifold, with $p(t, x, y), \mu(d x)$, and $B(x, r)$ as above. In addition, area $(d x)$ denotes the $(m-1)$-dimensional Hausdorffmeasure on $M$. There hold the following facts:

(i) (Localized heat kernel bounds): For every $r>0, x^{\prime} \in M$, there exist constants $A_{j}>0$ which only depend on $m$ and a lower bound of Ric in $B\left(x^{\prime}, 2 r\right)$, such that for all $(t, x, y) \in\left(0, r^{2}\right) \times B\left(x^{\prime}, r\right) \times B\left(x^{\prime}, r\right)$ one has

$$
\begin{aligned}
& e^{-A_{1} t} \mu(B(x, \sqrt{t}))^{-1 / 2} \mu(B(y, \sqrt{t}))^{-1 / 2} e^{-A_{2} \frac{d(x, y)^{2}}{t}} \\
& \leq p(t, x, y) \\
& \leq e^{A_{3} t} \mu(B(x, \sqrt{t}))^{-1 / 2} \mu(B(y, \sqrt{t}))^{-1 / 2} e^{-A_{4} \frac{d(x, y)^{2}}{t}} .
\end{aligned}
$$

(ii) (Local Bishop-Gromov estimate) For every $r>0, x \in M$, one has

$$
\mu(B(x, s)) \leq\left|\mathbb{S}^{m}\right| s^{m} e^{\sqrt{(m-1) K} s} \quad \text { for all } 0<s<2 r,
$$

and

$$
\text { area }(\{z \in M: d(z, x)=s\}) \leq\left|\mathbb{S}^{m}\right| s^{m-1} e^{\sqrt{(m-1) K} s} \quad \text { for all } 0<s<2 r,
$$

where $K \geq 0$ is any lower bound of Ric $\geq-K$ in $B(x, 2 r)$. 
(iii) (Local volume doubling property) For every $r>0, x \in M$, one has

$$
\mu(B(x, s)) \leq \mu\left(B\left(x, s^{\prime}\right)\right)\left(s / s^{\prime}\right)^{m} e^{\sqrt{(m-1) K} s} \quad \text { for all } 0<s^{\prime}<s<2 r
$$

where again $K \geq 0$ is any number such that $\mathrm{Ric} \geq-K$ in $B(x, 2 r)$.

The asserted heat kernel bound is the content of Theorem 6.1 in Saloff-Coste (1992). All remaining results follow from estimating the Riemannian volume density in exponential polar coordinates away from the cut-locus of $x$, and it is wellknown that this density can be controlled by the Laplacian of the distance function. Now the claims follow from observing that the Laplacian comparison theorem can be localized (as has been observed in Remark 2.6 in Pigola et al., 2008; see also inequality (1), (2) in Saloff-Coste, 1992).

\section{References}

S. Aida. Logarithmic derivatives of heat kernels and logarithmic Sobolev inequalities with unbounded diffusion coefficients on loop spaces. J. Funct. Anal. 174 (2), 430-477 (2000). MR1768982.

M. Arnaudon and A. Thalmaier. Li-Yau type gradient estimates and Harnack inequalities by stochastic analysis. In Probabilistic approach to geometry, volume 57 of Adv. Stud. Pure Math., pages 29-48. Math. Soc. Japan, Tokyo (2010). MR2605409.

C. Bär and F. Pfäffle. Wiener measures on Riemannian manifolds and the FeynmanKac formula. Mat. Contemp. 40, 37-90 (2011). MR3098046.

J.-M. Bismut. The Atiyah-Singer theorems: a probabilistic approach. I. The index theorem. J. Funct. Anal. 57 (1), 56-99 (1984a). MR744920.

J.-M. Bismut. Large deviations and the Malliavin calculus, volume 45 of Progress in Mathematics. Birkhäuser Boston, Inc., Boston, MA (1984b). ISBN 0-81763220-4. MR755001.

B. K. Driver. A Cameron-Martin type quasi-invariance theorem for pinned Brownian motion on a compact Riemannian manifold. Trans. Amer. Math. Soc. 342 (1), 375-395 (1994). MR1154540.

F.-Z. Gong and F.-Y. Wang. Heat kernel estimates with application to compactness of manifolds. Q. J. Math. 52 (2), 171-180 (2001). MR1838361.

B. Güneysu. Multiplicative matrix-valued functionals and the continuity properties of semigroups corresponding to partial differential operators with matrix-valued coefficients. J. Math. Anal. Appl. 380 (2), 709-725 (2011). MR2794426.

B. Güneysu. On generalized Schrödinger semigroups. J. Funct. Anal. 262 (11), 4639-4674 (2012). MR2913682.

B. Güneysu. Covariant Schrödinger semigroups on Riemannian manifolds, volume 264 of Operator Theory: Advances and Applications. Birkhäuser/Springer, Cham (2017). ISBN 978-3-319-68902-9; 978-3-319-68903-6. MR3751359.

W. Hackenbroch and A. Thalmaier. Stochastische Analysis. Mathematische Leitfäden. [Mathematical Textbooks]. B. G. Teubner, Stuttgart (1994). ISBN 3-519-02229-X. MR1312827.

E. P. Hsu. Brownian bridges on Riemannian manifolds. Probab. Theory Related Fields 84 (1), 103-118 (1990). MR1027823. 
E. P. Hsu. Stochastic analysis on manifolds, volume 38 of Graduate Studies in Mathematics. American Mathematical Society, Providence, RI (2002). ISBN 0-8218-0802-8. MR1882015.

X.-M. Li. On hypoelliptic bridge. Electron. Commun. Probab. 21, Paper No. 24, 12 (2016). MR3485393.

S. Pigola, M. Rigoli and A. G. Setti. Vanishing and finiteness results in geometric analysis, volume 266 of Progress in Mathematics. Birkhäuser Verlag, Basel (2008). ISBN 978-3-7643-8641-2. MR2401291.

L. Saloff-Coste. Uniformly elliptic operators on Riemannian manifolds. J. Differential Geom. 36 (2), 417-450 (1992). MR1180389.

A.-S. Sznitman. Brownian motion, obstacles and random media. Springer Monographs in Mathematics. Springer-Verlag, Berlin (1998). ISBN 3-540-64554-3. MR1717054.

A. Thalmaier and F.-Y. Wang. Gradient estimates for harmonic functions on regular domains in Riemannian manifolds. J. Funct. Anal. 155 (1), 109-124 (1998). MR1622800. 